\title{
Combining QCD and Electroweak Corrections to $W$-Boson Production at Hadron Colliders
}

\author{
G. Balossini ${ }^{1}$, C.M. Carloni Calame ${ }^{2}$, G. Montagna ${ }^{1}$, \\ M. Moretti ${ }^{3}$, O. Nicrosini ${ }^{4}$, M. Treccani ${ }^{3}$, A. Vicini ${ }^{5}$ \\ 1-Dipartimento di Fisica Nucleare e Teorica, Università di Pavia, and INFN, Sezione di Pavia \\ 2-School of Physics and Astronomy, Southampton University, UK \\ 3-Dipartimento di Fisica, Università di Ferrara, and INFN, Sezione di Ferrara, Italy \\ 4- INFN, Sezione di Pavia, and Dipartimento di Fisica Nucleare e Teorica, Università di Pavia \\ 5- Dipartimento di Fisica, Università di Milano, and INFN, Sezione di Milano
}

\begin{abstract}
We review the present status of higher-order calculations for single $W$ and $Z$ boson production at hadron colliders, and present some preliminary results on the combination of electroweak and QCD corrections to a sample of observables of the process $\stackrel{(-)}{p p} \rightarrow$ $W^{ \pm} \rightarrow \mu^{ \pm}+X$ at the Tevatron and at the LHC. Our phenomenological analysis shows that a high-precision knowledge of QCD and a careful combination of electroweak and strong contributions is mandatory in view of the anticipated experimental accuracy.
\end{abstract}

\section{Higher-order QCD/electroweak calculations and tools}

The Drell-Yan process has played a key role in the development of our understandig of $\mathrm{QCD}$ and electroweak (EW) interactions in hadron collider experiments, both from the experimental and theoretical point of view $[2,3]$. Concerning QCD calculations and tools for EW gauge boson production at hadron colliders, the present situation reveals a quite rich structure, that includes next-to-leading-order (NLO) and next-tonext-to-leading-order (NNLO) corrections to $W / Z$ total production rate $[4,5]$, NLO calculations for $W, Z+1,2$ jets signatures $[6,7]$ (available in the codes DYRAD and MCFM), resummation of leading and nextto-leading logarithms due to soft gluon radiation $[8,9]$ (implemented in the Monte Carlo ResBos), NLO corrections merged with QCD Parton Shower (PS) evolution

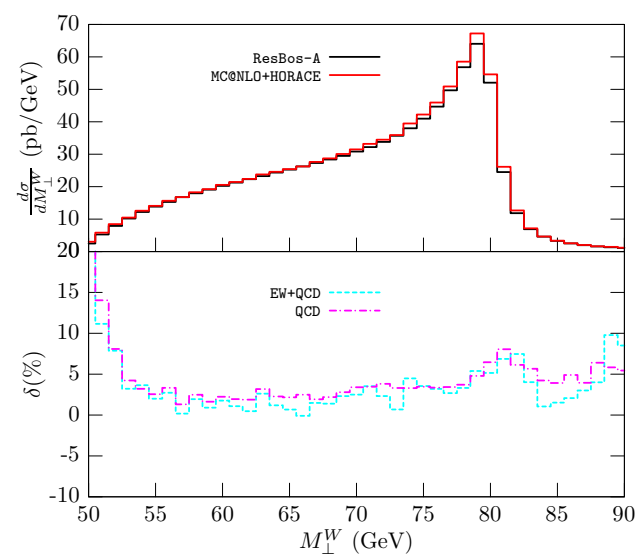

Figure 1: $W$ transverse mass distribution at the Tevatron. Percent differences w.r.t. $\operatorname{Resbos}(-\mathrm{A})$. [10] (in the event generator MC@NLO), NNLO corrections to $W / Z$ production in fully differential form $[11,12,13,14]$ (available in the Monte Carlo program FEWZ), as well as leading-order multi-parton matrix elements generators matched with vetoed PS, such as, for instance, ALPGEN [15], MADEVENT [16] and SHERPA [17]. As far as complete $\mathcal{O}(\alpha)$ EW corrections to Drell-Yan processes are concerned, they have been computed independently by various authors in $[18,19,20,21,22]$ 
for $W$ production and in [23] for $Z$ production. EW tools implementing exact NLO corrections to $W$ production are DK [18], WGRAD2 [19], SANC [21] and HORACE [22], while ZGRAD2 [23] includes the full set of $\mathcal{O}(\alpha)$ EW corrections to $Z$ production. The predictions of a subset of such calculations have been recently compared, at the level of same input parameters and cuts, in the proceedings of the Les Houches [24] and TEV4LHC [25] workshops for $W$ production, finding a very satisfactory agreement between the various, independent calculations. The effect of the EW corrections on the determination of the $W$ mass is large and is dominated by final-state QED radiation, enhanced by large collinear logarithms. NLO EW corrections induce a shift in the extracted value of $m_{W}$ of the order of $100 \mathrm{MeV}$ and higher-order effects contribute with a further shift of $\sim 10 \%$ of the NLO contribution with opposite sign [26]; the latter can not be neglected, in view of the present accuracy reached at the Tevatron (see the talk by S. Malik [27]) and foreseen at the LHC $\left(\Delta m_{W} \sim 15 \mathrm{MeV}\right)$. In spite of this detailed knowledge of higher-order EW and QCD corrections, the combination of their effects is still at a very preliminary stage. There is only one attempt known in the literature [28], where the effects of QCD resummation are combined with NLO QED final-state corrections, leaving room for more detailed studies of the interplay between EW and QCD corrections to $W / Z$ production at hadron colliders.

\section{Combining QCD and EW corrections}

The combination of QCD and EW corrections, using a factorized expression for the mixed contributions, can be cast in the following form:

$$
\left[\frac{d \sigma}{d \mathcal{O}}\right]_{Q C D \oplus E W}=\left\{\frac{d \sigma}{d \mathcal{O}}\right\}_{Q C D}+\left\{\left[\frac{d \sigma}{d \mathcal{O}}\right]_{E W}-\left[\frac{d \sigma}{d \mathcal{O}}\right]_{B o r n}\right\}
$$

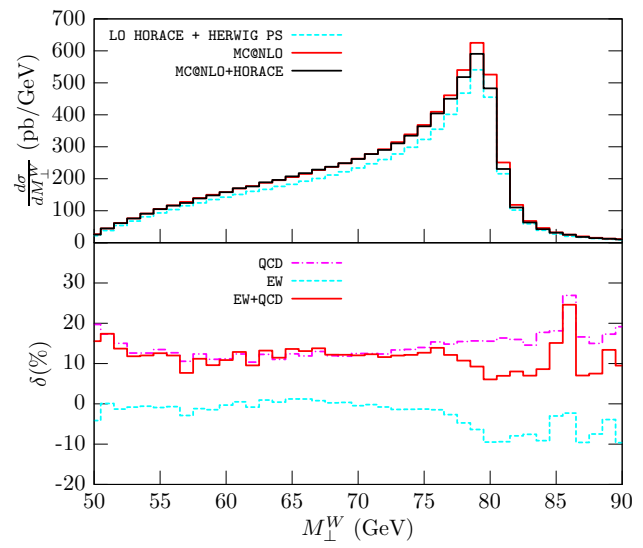

Figure 2: $W$ transverse mass distribution at the LHC. Percent differences w.r.t. LO+PS results where $d \sigma / d \mathcal{O}_{Q C D}$ stands for the prediction of the observable $d \sigma / d \mathcal{O}$, as obtained by means of one of the state-of-the-art generators available in the literature, $d \sigma / d \mathcal{O}_{E W}$ is the HORACE prediction for the EW corrections to the $d \sigma / d \mathcal{O}$ observable, and $d \sigma / d \mathcal{O}_{\text {Born }}$ is the lowest-order result for the observable of interest. The label HERWIG PS in the second term in r.h.s. of Eq. (1) means that EW corrections are convoluted with QCD PS evolution through the HERWIG event generator, in order to (approximately) include mixed $\mathcal{O}\left(\alpha \alpha_{s}\right)$ corrections and to obtain a more realistic description of the observables under study. Actually, since the QCD shower evolution generates partons in the soft/collinear approximation, the results obtained for $\mathcal{O}\left(\alpha \alpha_{s}\right)$ corrections according to such a procedure are expected to be unreliable when hard non-collinear QCD radiation turns out to be important. However, beyond this approximation, a full two-loop calculation of $\mathcal{O}\left(\alpha \alpha_{s}\right)$ corrections, which is presently unavailable, would be required. 


\section{$3 \quad$ Numerical results}

The preliminary numerical results shown in the present Section have been obtained using as standard cuts $p_{\perp}^{l}, p_{\perp}^{\text {miss }}>25 \mathrm{GeV}$ for the minimum transverse momentum of the charged lepton and for the missing transverse momentum (both at the Tevatron and at the LHC) and $\eta_{\max }^{l}<1.2(2.5)$ at the Tevatron (LHC) for the maximum lepton pseudo-rapidity. We have also considered the case with the additional cut $M_{\perp}^{W}>$ $1 \mathrm{TeV}$ on the $W$ transverse mass, cfr. Fig.3, to study the region where new heavy gauge boson could be produced. A careful tuning procedure has been carried on, to check that all the programs used in the comparison yield the same numbers under equal conditions in terms of input parameters, cuts and perturbative order. We have chosen the $G_{\mu}$ input scheme for the calculation of EW corrections, where, in particular, the (effective) electromagnetic coupling constant is given in the tree-level approximation by $\alpha_{G_{\mu}}^{\text {tree }}=(\sqrt{2} / \pi) G_{\mu} m_{W}{ }^{2} \sin ^{2} \vartheta_{W}$. However, for the coupling of external photons to charged particles needed for the evaluation of photonic corrections we use $\alpha(0)=1 / 137.03599911$.

In Fig. 1 we plot the $W$ transverse mass distribution at the Tevatron and compare the results obtained with Resbos vs. MC@NLO (only QCD corrections) and those computed with Resbos-A vs. MC@NLO+HORACE (QCD+EW corrections). We observe an overall difference due to a different normalization of the two pure QCD codes and a clear deviation in the low transverse mass tail, which reaches the $15 \%$ level. The EW effects are dominated by QED final-state radiation; nevertheless the inclusion of exact $\mathcal{O}(\alpha)$ results and of higher-order QED terms is necessary, in view of the foreseen experimental accuracy. In Fig. 2 we plot the $W$ transverse mass distribution at the LHC and compare the LO+PS results with the pure QCD predictions of $\mathrm{MC} @ \mathrm{NLO}$ and with the QCD+EW combination of MC@NLO+HORACE as in Eq.(1). The QCD corrections are large and positive and compensate the effect of the EW corrections which are negative. We observe that the peaked shape of the EW effects (cfr. Fig.4 in ref.[22]) is broadened by the convolution with the QCD parton shower. The inclusion of both EW and QCD corrections is necessary to obtain the proper descritpion of the peak region and in particular the correct shape of the transverse mass distribution, which is relevant for the extraction of the $W$ mass. In Fig. 3 we plot the lepton transverse momentum distribution at the LHC and compare the LO+PS results with the pure QCD predictions of $\mathrm{MC} @ \mathrm{NLO}$ and with the QCD+EW combination of MC@NLO+HORACE. The large and negative EW corrections due to the presence of EW Sudakov logarithms sum up with large and negative effects due to the QCD corrections and reduce the distribution by $-30 \%$ to $-50 \%$ for $500 \leq p_{\perp}^{l} \leq 1000 \mathrm{GeV}$. The large mass tail of the distribution is relevant for all the searches of physics beyond the Standard Model (SM), in particular of new heavy gauge bosons which could decay into a pair of leptons. The 
accurate estimate of the SM background is very important to set reliable lower bounds on the masses of the new particles. In the last two examples we computed the relative effect of the various corrections w.r.t. the LO+PS results, having also observed the role of the QCD parton-shower in the simulation of the observables of interest.

In conclusion, we have presented the preliminary results of a study aiming at combining QCD and EW corrections, according to Eq.(1), in order to obtain an accurate description of the charged-current Drell-Yan process. The impact of the interplay between the two sets of corrections can not be neglected in data analysis, to match the accuracy foreseen e.g. in the measurement of the $W$ boson mass.

\section{References}

[1] Slides: http://indico. cern. ch/contributionDisplay py? contribId=176\&sessionId=6\&conf Id=9499

[2] P.M. Nadolsky, AIP Conf. Proc. 753 (2005) 158

[3] U. Baur, Electroweak physics at the Tevatron and LHC: Theoretical status and perspectives, arXiv:hep$\mathrm{ph} / 0511064$

[4] G. Altarelli, R. K. Ellis and G. Martinelli, Nucl. Phys. B157 (1979) 461

[5] R. Hamberg, W. L. van Neerven and T. Matsuura, Nucl. Phys. B359 (1991) 343 [Erratum Nucl. Phys. B644 (2002) 403]

[6] W.T. Giele, E.W.N. Glover and D.A. Kosower, Nucl. Phys. B403 (1993) 633

[7] J.M. Campbell and R.K. Ellis, Phys. Rev. D65 (2002) 113007

[8] C. Balazs and C. P. Yuan, Phys. Rev. D56 (1997) 5558

[9] F. Landry, R. Brock, P.M. Nadolsky and C.-P. Yuan, Phys. Rev. D67 (2003) 073016

[10] S. Frixione and B. R. Webber, JHEP 0206 (2002) 029

[11] C. Anastasiou, L. J. Dixon, K. Melnikov and F. Petriello, Phys. Rev. Lett. 91 (200 3 ) 182002

[12] C. Anastasiou, L. J. Dixon, K. Melnikov and F. Petriello, Phys. Rev. D69 (2004) 094008

[13] K. Melnikov and F. Petriello, Phys. Rev. Lett. 96 (2006) 231803

[14] K. Melnikov and F. Petriello, Phys. Rev. D74 (2006) 114017

[15] M.L. Mangano, M. Moretti, F. Piccinini, R. Pittau and A.D. Polosa, JHEP 0307 (2003) 001

[16] T. Stelzer and W.F. Long, Comp. Phys. Commun. 81 (1994) 357; F. Maltoni and T. Stelzer, JHEP 02 (2003) 027

[17] T. Gleisberg, S. Höche, F. Krauss, A. Schälicke, S. Schumann and J. Winter, JHEP 0402 (2004) 056

[18] S. Dittmaier and M. Krämer, Phys. Rev. D65 (2002) 0703007

[19] U. Baur and D. Wackeroth, Phys. Rev. D70 (2004) 073015

[20] V. A. Zykunov, Eur. Phys. J. Direct C3 (2001) 9; Phys. Atom. Nucl. 69 (2006) 1522

[21] A. Arbuzov, D. Bardin, S. Bondarenko, P. Christova, L. Kalinovskaya, G. Nanava and R. Sa dykov, Eur. Phys. J. C46 (2006) 407

[22] C.M. Carloni Calame, G. Montagna, O. Nicrosini and A. Vicini, JHEP 12 (2006) 016

[23] U. Baur, O. Brein, W. Hollik, C. Schappacher and D. Wackeroth, Phys. Rev. D65 (2002) 033007

[24] C. Buttar et al., arXiv:hep-ph/0604120

[25] C. E. Gerber et al. [The TeV4LHC-Top and Electroweak Working Group], arXiv:0705.3251 [hep-ph].

[26] C.M. Carloni Calame, G. Montagna, O. Nicrosini and M. Treccani, Phys. Rev. D69 (2004) 037301

[27] Slides: http://indico.cern.ch/contributionDisplay.py?contribId=105\&sessionId=9\&conf Id=9499

[28] Q.-H. Cao and C.-P. Yuan, Phys. Rev. Lett. 93 (2004) 042001; arXiv:hep-ph/0401171 